\title{
ON VOLTERRA AND ORTHOGONALITY PRESERVING QUADRATIC STOCHASTIC OPERATORS
}

\author{
FARRUKH MUKHAMEDOV AND MUHAMMAD HAFIZUDDIN BIN MOHD TAHA
}

Received 30 December, 2013

\begin{abstract}
A quadratic stochastic operator (in short QSO) is usually used to present the time evolution of differing species in biology. Some quadratic stochastic operators have been studied by Lotka and Volterra. In the present paper, we first give a simple characterization of Volterra QSO in terms of absolutely continuity of discrete measures. Moreover, we provide its generalization in continuous setting. Further, we introduce a notion of orthogonal preserving QSO, and describe such kind of operators defined on two dimensional simplex. It turns out that orthogonal preserving QSOs are permutations of Volterra QSO. The associativity of genetic algebras generated by orthogonal preserving QSO is studied too.
\end{abstract}

2010 Mathematics Subject Classification: 37E99; 37N25; 39B82; 47H60; 92D25

Keywords: quadratic stochastic operator, Volterra operator, orthogonal preserving

\section{INTRODUCTION}

The history of quadratic stochastic operators (QSO) can be traced back to Bernstein's work [1] where such kind of operators appeared from the problems of population genetics (see also [9]). Such kind of operators describe time evolution of variety species in biology are represented by so-called Lotka-Volterra(LV) systems [8,26,27].

A quadratic stochastic operator is usually used to present the time evolution of species in biology, which arises as follows. Consider a population consisting of $m$ species (or traits) $1,2, \cdots, m$. We denote a set of all species (traits) by $I=\{1,2, \cdots, m\}$. Let $x^{(0)}=\left(x_{1}^{(0)}, \cdots, x_{m}^{(0)}\right)$ be a probability distribution of species at an initial state and $P_{i j, k}$ be a probability that individuals in the $i^{t h}$ and $j^{t h}$ species (traits) interbreed to produce an individual from $k^{t h}$ species (trait). Then a probability distribution $x^{(1)}=\left(x_{1}^{(1)}, \cdots, x_{m}^{(1)}\right)$ of the spices (traits) in the first generation can be found

The first author acknowledges the Junior Associate scheme of the Abdus Salam International Centre for Theoretical Physics, Trieste, Italy. 
as a total probability, i.e.,

$$
x_{k}^{(1)}=\sum_{i, j=1}^{m} P_{i j, k} x_{i}^{(0)} x_{j}^{(0)}, \quad k=\overline{1, m} .
$$

This means that the association $x^{(0)} \rightarrow x^{(1)}$ defines a mapping $V$ called the evolution operator. The population evolves by starting from an arbitrary state $x^{(0)}$, then passing to the state $x^{(1)}=V\left(x^{(0)}\right)$ (the first generation), then to the state $x^{(2)}=$ $V\left(x^{(1)}\right)=V\left(V\left(x^{(0)}\right)\right)=V^{(2)}\left(x^{(0)}\right)$ (the second generation), and so on. Therefore, the evolution states of the population system described by the following discrete dynamical system

$$
x^{(0)}, \quad x^{(1)}=V\left(x^{(0)}\right), \quad x^{(2)}=V^{(2)}\left(x^{(0)}\right), \quad x^{(3)}=V^{(3)}\left(x^{(0)}\right) \cdots
$$

In other words, a QSO describes a distribution of the next generation if the distribution of the current generation was given. The fascinating applications of QSO to population genetics were given in [9]. Furthermore, the quadratic stochastic operator was considered an important source of analysis for the study of dynamical properties and modelings in various fields such as biology [6, 7, 10, 11, 18], physics [20, 23], economics and mathematics [3, 9, 23-25].

In [5], it was given along self-contained exposition of the recent achievements and open problems in the theory of the QSO. The main problem in the nonlinear operator theory is to study the behavior of nonlinear operators. This problem was not fully finished even in the class of QSO (the QSO is the simplest nonlinear operator). The difficulty of the problem depends on the given cubic matrix $\left(P_{i j k}\right)_{i, j, k=1}^{m}$. An asymptotic behavior of the QSO even on the small dimensional simplex is complicated [12, 17, 25,29].

In the present paper, we first give a simple characterization of Volterra QSO (see [3]) in terms of absolutely continuity of discrete measures (see Section 3). Further, in Section 4 we introduce a notion of orthogonal preserving QSO, and describe such kind of operators defined on two dimensional simplex. It turns out that orthogonal preserving QSOs are permutations of Volterra QSO. In Section 5, we study associativity of genetic algebras generated by orthogonal preserving QSO.

\section{PReliminaries}

An evolutionary operator of a free population is a (quadratic) mapping of the simplex

$$
S^{m-1}=\left\{\mathbf{x}=\left(x_{1}, \ldots, x_{m}\right) \in \mathbb{R}^{m} \mid x_{i} \geq 0, \sum_{i=1}^{m} x_{i}=1\right\}
$$


into itself of the form

$$
V: x_{k}^{\prime}=\sum_{i, j=1}^{m} P_{i j, k} x_{i} x_{j}, \quad k=1,2, \ldots, m
$$

where $P_{i j, k}$ are coefficient of heredity and

$$
P_{i j, k} \geq 0, \quad P_{i j, k}=P_{j i, k}, \quad \sum_{k=1}^{m} P_{i j, k}=1, i, j, k=1,2, \ldots, m
$$

Such a mapping is called quadratic stochastic operator (QSO).

Note that every element $\mathbf{x} \in S^{m-1}$ is a probability distribution on $I=\{1, \ldots, m\}$. The population evolves starting from an arbitrary initial state $\mathbf{x} \in S^{m-1}$ (probability distribution on $E$ ) to the state $\mathbf{x}^{\prime}=V(\mathbf{x})$ in the next generation, then to the state $\mathbf{x}^{\prime \prime}=V^{2}(\mathbf{x})=V(V(\mathbf{x}))$, and so on.

For a given $\mathbf{x}^{(0)} \in S^{m-1}$, the trajectory

$$
\left\{\mathbf{x}^{(n)}\right\}, n=0,1,2, \ldots
$$

of $\mathbf{x}^{(0)}$ under the action of QSO (2.2) is defined by

$$
\mathbf{x}^{n+1}=V\left(\mathbf{x}^{(n)}\right), \quad n=0,1,2, \ldots
$$

A QSO $V$ defined by (2.2) is called Volterra operator [3] if one has

$$
P_{i j, k}=0 \text { if } k \notin\{i, j\}, \forall i, j, k \in I .
$$

Note that it is obvious that the biological behavior of condition (2.4) is that the offspring repeats one of its parents' genotype (see [3,5]).

Definition 1. Let $\mathbf{x}=\left(x_{1}, \ldots, x_{m}\right)$ and $\mathbf{y}=\left(y_{1}, \ldots, y_{m}\right)$. We say that $\mathbf{x}$ is equivalent to $\mathbf{y}(\mathbf{x} \sim \mathbf{y})$ if

(i) $\mathbf{x} \prec \mathbf{y}$ ( $\mathbf{x}$ is absolutely continuous with respect to $\mathbf{y}$ ) if $y_{k}=0 \Rightarrow x_{k}=0$,

(ii) $\mathbf{y} \prec \mathbf{x}$ if $x_{k}=0 \Rightarrow y_{k}=0$.

Definition 2. Let $I=\{1,2, \ldots, m\}$ and $\operatorname{Supp}(\mathbf{x})=\left\{i \in I \mid x_{i} \neq 0\right\}$. Then $\mathbf{x}$ is singular or orthogonal to $\mathbf{y}(\mathbf{x} \perp \mathbf{y})$ if $\operatorname{Supp}(\mathbf{x}) \cap \operatorname{Supp}(\mathbf{y})=\varnothing$.

Note that if $\mathbf{x} \perp \mathbf{y} \Rightarrow \mathbf{x} \cdot \mathbf{y}=0$, whenever $\mathbf{x}, \mathbf{y} \in S^{m-1}$. Here $\mathbf{x} \cdot \mathbf{y}$ stands for the usual scalar product in $\mathbb{R}^{m}$.

\section{ON VOLTERRA QSO}

In this section we are going to give a characterization of Volterra quadratic operator in terms of the above given order. Note that dynamics of Volterra QSO was investigated in [3]. Certain other properties of such kind of operators has been studied in [13]. Some generalizations of Volterra QSO were studied in [14, 21,22].

Recall that the vertices of the simplex $S^{m-1}$ are described by the elements $e_{k}=$ $\left(\delta_{1 k}, \delta_{2 k}, \ldots, \delta_{m k}\right)$, where $\delta_{i k}$ is the Kronecker's delta. 
Theorem 1. Let $V: S^{m-1} \rightarrow S^{m-1}$ be a $Q S O$. Then the following conditions are equivalent:

(i) $V$ is a Volterra QSO;

(ii) one has $V(\mathbf{x}) \prec \mathbf{x}$ for all $\mathbf{x} \in S^{m-1}$.

Proof. (i) $\Rightarrow$ (ii). It is known ([3]) that any Volterra QSO can be represented as follows:

$$
(V(x))_{k}=x_{k}\left(1+\sum_{i=1}^{m} a_{k i} x_{i}\right), k=\overline{1, m}
$$

where $a_{k i}=-a_{i k},\left|a_{k i}\right| \leq 1$.

From the equality we immediately get $V(\mathbf{x}) \prec \mathbf{x}$ for all $\mathbf{x} \in S^{m-1}$.

(ii) $\Rightarrow$ (i). Let $\mathbf{x}=e_{k},(k \in\{1, \ldots, m\})$. Then due to $V(\mathbf{x}) \prec \mathbf{x}$ from (2.2) one finds

$$
P_{k k, k}=1 \quad P_{k k, i}=0, i \neq k .
$$

Now assume that $\mathbf{x}=\lambda e_{i}+(1-\lambda) e_{j}$, where $\lambda \in(0,1)$. Let $k \notin\{i, j\}$, then from (2.2) one finds that

$$
V(\mathbf{x})_{k}=P_{i i, k} \lambda^{2}+2 \lambda(1-\lambda) P_{i j, k}+P_{j j, k}(1-\lambda)^{2}
$$

Taking into account (3.2) and the relation $V(\mathbf{x}) \prec \mathbf{x}$ with (3.3) one gets $P_{i j, k}=0$. This completes the proof.

The proved theorem characterizes Volterra QSO in terms of absolute continuity of distributions. Therefore, this theorem will allow to define such kind of operators in abstract settings. Let us demonstrate it.

Assume that $(E, \mathcal{F})$ be a measurable space and $S(E, \mathcal{F})$ be the set of all probability measures on $(E, \mathcal{F})$.

Recall that a mapping $V: S(E, \mathcal{F}) \rightarrow S(E, \mathcal{F})$ is called a quadratic stochastic operator $(Q S O)$ if, for an arbitrary measure $\lambda \in S(E, \mathcal{F})$ the measure $\lambda^{\prime}=V(\lambda)$ is defined as follows

$$
\lambda^{\prime}(A)=\int_{E} \int_{E} P(x, y, A) d \lambda(x) d \lambda(y), \quad A \in \mathcal{F},
$$

where $P(x, y, A)$ satisfies the following conditions:

(i) $P(x, y, \cdot) \in S(E, \mathcal{F})$ for any fixed $x, y \in E$;

(ii) For any fixed $A \in \mathscr{F}$ the function $P(x, y, A)$ is measurable of two variables $x$ and $y$ on $(E \times E, \mathcal{F} \otimes \mathcal{F})$;

(iii) the function $P(x, y, A)$ is symmetric, i.e. $P(x, y, A)=P(y, x, A)$ for any $x, y \in E$ and $A \in \mathscr{F}$.

Note that when $E$ is finite, i.e. $E=\{1, \ldots, m\}$, then a QSO on $S(E, \mathcal{F})=S^{m-1}$ is defined as in (2.2) with $P_{i j, k}=P(i, j, k)$. 
We recall that a measure $\mu \in S(E, \mathcal{F})$ is absolutely continuous w.r.t. a measure $v \in S(E, \mathcal{F})$ if $\nu(A)=0$ implies $\mu(A)=0$, and they are denoted by $\mu \prec \nu$. Put

$$
\operatorname{null}(\mu)=\bigcup_{\mu(A)=0} A
$$

Then the support of the measure $m$ is defined by $\operatorname{supp}(\mu)=E \backslash$ null $(\mu)$. Two measures $\mu, v \in S(E, \mathcal{F})$ are called singular if $\operatorname{supp}(\mu) \cap \operatorname{supp}(\nu)=\varnothing$, and they are denoted by $\mu \perp v$.

Definition 3. A QSO given by (3.4) is called Volterra if $V \lambda \prec \lambda$ for all $\lambda \in$ $S(E, \mathcal{F})$.

Theorem 2. Let $V$ be given by (3.4). Then $V$ is Volterra $Q S O$ if and only if $P(x, y, A)=0$ for all $x, y \notin A$.

Proof. First we assume that $V$ is Volterra QSO. Take any $x, y \in E$ and consider the measure $v=\frac{1}{2}\left(\delta_{x}+\delta_{y}\right)$, where $\delta_{x}$ is a delta-measure, i.e. $\delta_{x}(A)=\chi_{A}(x)$. Then from (3.4) one finds that

$$
V(v)(A)=\frac{1}{4}(P(x, x, A)+P(y, y, A)+2 P(x, y, A)) .
$$

From $V v \prec v$ and $v(A)=0$ (if $x, y \notin A$ ) we infer that $V(v)(A)=0$, this yields that $P(x, x, A)=P(y, y, A)=P(x, y, A)=0$ if $x, y \notin A$.

Let us suppose that $P(x, y, A)=0$ is valid for all $x, y \notin A$. Assume that for $\mu \in S(E, \mathcal{F})$ one has $\mu(B)=0$ for some $B \in \mathcal{F}$. Let us show that $V(\mu)(B)=0$. Indeed, from (3.4) and the conditions one gets

$$
\begin{aligned}
V(\mu)(B)= & \int_{E} \int_{E} P(x, y, B) d \mu(x) d \mu(y) \\
= & \int_{E \backslash B} \int_{E \backslash B} P(x, y, B) d \mu(x) d \mu(y)+\int_{E \backslash B} \int_{B} P(x, y, B) d \mu(x) d \mu(y) \\
& +\int_{B} \int_{E \backslash B} P(x, y, B) d \mu(x) d \mu(y)+\int_{B} \int_{B} P(x, y, B) d \mu(x) d \mu(y) \\
= & \int_{E \backslash B} \int_{E \backslash B} P(x, y, B) d \mu(x) d \mu(y)=0
\end{aligned}
$$

This completes the proof.

\section{ORThOgonal PRESERVING(OP) QSO IN 2D SimPleX}

We recall that two vectors $\mathbf{x}$ and $\mathbf{y}$ belonging to $S^{m-1}$ are called singular or orthogonal if $\mathbf{x} \cdot \mathbf{y}=0$.

A mapping $V: S^{m-1} \rightarrow S^{m-1}$ is called orthogonal preserving (O.P.) if one has $V(\mathbf{x}) \perp V(\mathbf{y})$ whenever $\mathbf{x} \perp \mathbf{y}$. 
In this section, we are going to describe orthogonal preserving QSO defined in 2D simplex.

Let us assume that $V: S^{2} \rightarrow S^{2}$ be an orthogonal preserving QSO.

This means that

$$
V(1,0,0) \perp V(0,1,0) \perp V(0,0,1)
$$

Now from the definition of QSO (see (2.2)), we immediately get

$$
\left(P_{11,1}, P_{11,2}, P_{11,3}\right) \perp\left(P_{22,1}, P_{22,2}, P_{22,3}\right) \perp\left(P_{33,1}, P_{33,2}, P_{33,3}\right)
$$

Since in the simplex $S^{2}$ there are 3 orthogonal vectors which are

$$
(1,0,0),(0,1,0),(0,0,1) \text {. }
$$

We conclude the vectors

$$
\left(P_{11,1}, P_{11,2}, P_{11,3}\right),\left(P_{22,1}, P_{22,2}, P_{22,3}\right), \quad\left(P_{33,1}, P_{33,2}, P_{33,3}\right)
$$

could be permutation of the given orthogonal vectors. Therefore we have 6 possibilities and we consider each of these possibilities one by one.

Let us first assume that

$$
\begin{array}{lll}
P_{11,1}=0 & P_{11,2}=0 & P_{11,3}=1 \\
P_{22,1}=0 & P_{22,2}=1 & P_{22,3}=0 \\
P_{33,1}=1 & P_{33,2}=0 & P_{33,3}=0
\end{array}
$$

Now our aim is to find some conditions for the others coefficients of the given QSO. Let us consider the following vectors

$$
\mathbf{x}=\left(\frac{1}{2}, \frac{1}{2}, 0\right) \quad \mathbf{y}=(0,0,1)
$$

which are clearly orthogonal. One can see that

$$
\begin{aligned}
& V(\mathbf{x})=\frac{1}{4}\left(2 P_{12,1}, 2 P_{12,2}+1,1+2 P_{12,3}\right) \\
& V(\mathbf{y})=(1,0,0)
\end{aligned}
$$

Therefore, the orthogonal preservably of $V$ yields $P_{12,1}=0$. From $\sum_{i=1}^{3} P_{12, i}=1$ one gets

$$
P_{12,2}+P_{12,3}=1
$$

Now consider

$$
\mathbf{x}=\left(0, \frac{1}{2}, \frac{1}{2}\right) \quad \mathbf{y}=(1,0,0)
$$

Then we have

$$
\begin{aligned}
& V(\mathbf{x})=\frac{1}{4}\left(2 P_{23,1}+1,2 P_{23,2}+1,2 P_{23,3}\right), \\
& V(\mathbf{y})=(0,0,1)
\end{aligned}
$$


Again the orthogonal preservability of $V$ implies $P_{23,3}=0$ and hence we get

$$
P_{23,1}+P_{23,2}=1
$$

Finally, let us take

$$
\mathbf{x}=\left(\frac{1}{2}, 0, \frac{1}{2}\right) \quad \mathbf{y}=(0,1,0)
$$

Then one has

$$
\begin{aligned}
& V(\mathbf{x})=\frac{1}{4}\left(1+2 P_{13,1}, 2 P_{13,2}, 1+2 P_{13,3}\right), \\
& V(\mathbf{y})=(0,1,0)
\end{aligned}
$$

Hence, we conclude that $P_{13,2}=0$, which implies

$$
P_{13,1}+P_{13,3}=1
$$

Taking into account the obtained equations, we denote

$$
P_{12,2}=\alpha \quad P_{23,1}=\beta \quad P_{13,1}=\gamma
$$

Correspondingly, one finds

$$
P_{12,3}=1-\alpha \quad P_{23,2}=1-\beta \quad P_{13,3}=1-\gamma
$$

Therefore, $V$ has the following form

$$
V_{\alpha, \beta, \gamma}^{(1)}:\left\{\begin{array}{l}
x^{\prime}=z^{2}+2 \gamma x z+2 \beta y z \\
y^{\prime}=y^{2}+2 \alpha x y+2(1-\beta) y z \\
z^{\prime}=x^{2}+2(1-\alpha) x y+2(1-\gamma) x z
\end{array}\right.
$$

Similarly, considering all other possibilities we obtain the following operators:

$$
\begin{aligned}
& V_{\alpha, \beta, \gamma}^{(2)}:\left\{\begin{array}{l}
x^{\prime}=x^{2}+2 \alpha x y+2 \gamma x z \\
y^{\prime}=y^{2}+2(1-\alpha) x y+2 \beta y z \\
z^{\prime}=z^{2}+2(1-\gamma) x z+2(1-\beta) y z
\end{array}\right. \\
& V_{\alpha, \beta, \gamma}^{(3)}:\left\{\begin{array}{l}
x^{\prime}=x^{2}+2 \alpha x y+2 \gamma x z \\
y^{\prime}=z^{2}+2(1-\gamma) x z+2 \beta y z \\
z^{\prime}=y^{2}+2(1-\alpha) x y+2(1-\beta) y z
\end{array}\right. \\
& V_{\alpha, \beta, \gamma}^{(4)}:\left\{\begin{array}{l}
x^{\prime}=y^{2}+2 \alpha x y+2 \beta y z \\
y^{\prime}=z^{2}+2 \gamma x z+2(1-\beta) y z \\
z^{\prime}=x^{2}+2(1-\alpha) x y+2(1-\gamma) x z
\end{array}\right. \\
& V_{\alpha, \beta, \gamma}^{(5)}:\left\{\begin{array}{l}
x^{\prime}=y^{2}+2 \alpha x y+2 \beta y z \\
y^{\prime}=x^{2}+2(1-\alpha) x y+2 \gamma x z \\
z^{\prime}=z^{2}+2(1-\gamma) x z+2(1-\beta) y z
\end{array}\right. \\
& V_{\alpha, \beta, \gamma}^{(6)}:\left\{\begin{array}{l}
x^{\prime}=z^{2}+2 \gamma x z+2 \beta y z \\
y^{\prime}=x^{2}+2 \alpha x y+2(1-\gamma) x z \\
z^{\prime}=y^{2}+2(1-\alpha) x y+2(1-\beta) y z
\end{array}\right.
\end{aligned}
$$


So, if $V$ is OP QSO, then it can be one of the above given operators. Now we are going to show the obtained operators are indeed orthogonal preserving.

Theorem 3. Let $V$ be an orthogonal preserving $Q S O$ on $S^{2}$. Then $V$ has one of the following forms:

$$
V_{\alpha, \beta, \gamma}^{(1)}, V_{\alpha, \beta, \gamma}^{(2)}, V_{\alpha, \beta, \gamma}^{(3)}, V_{\alpha, \beta, \gamma}^{(4)}, V_{\alpha, \beta, \gamma}^{(5)}, V_{\alpha, \beta, \gamma}^{(6)}
$$

Proof. According to the calculations done above, we have six listed operators. Now we show that these operators are indeed orthogonal preserving. Without loss of generality, we may consider operator $V_{\alpha, \beta, \gamma}^{(1)}$.

Assume that $\mathbf{x} \perp \mathbf{y}$. Then we have the following possibilities:

$$
\mathbf{x} \perp \mathbf{y} \Longleftrightarrow \begin{cases}\mathbf{x}=(x, y, 0) & \mathbf{y}=(0,0,1), \\ \mathbf{x}=(x, 0, z) & \mathbf{y}=(0,1,0), \\ \mathbf{x}=(0, y, z) & \mathbf{y}=(1,0,0)\end{cases}
$$

Let $\mathbf{x}=(x, y, 0)$ and $\mathbf{y}=(0,0,1)$. Then one gets

$$
V_{\alpha, \beta, \gamma}^{(1)}(\mathbf{x})=\left(0, y^{2}+2 \alpha x y, x^{2}+2(1-\alpha) x y\right), \quad V_{\alpha, \beta, \gamma}^{(1)}(\mathbf{y})=(1,0,0) .
$$

It is clear there are orthogonal. By the same argument, for other two cases, we can establish the orthogonality of $V_{\alpha, \beta, \gamma}^{(1)}(\mathbf{x})$ and $V_{\alpha, \beta, \gamma}^{(1)}(\mathbf{y})$. This completes the proof.

Remark 1. We note that the operators given in (4.2) are permutations of Volterra QSO. In [4] it was proved that permutations of Volterra operators are automorphisms of the simplex.

Remark 2. It is well-known that linear stochastic operators are orthogonal preserving if and only if they are permutations of the simplex. We point out that if $\alpha=\beta=\gamma=1 / 2$, then the operators (4.2) reduce to such kind of permutations.

To investigate dynamics of the obtained operators (see (4.2)), it is natural to employ the conjugacy of operators.

Recall that two QSO $V^{(1)}$ and $V^{(2)}$ are said to be conjugate if there exists a permutation $T_{\pi}:(x, y, z) \rightarrow(\pi(x), \pi(y), \pi(z))$ such that $T_{\pi}^{-1} V^{(1)} T_{\pi}=V^{(2)}$ and we denote this by $V^{(1)} \sim^{\pi} V^{(2)}$.

In our setting, we need to consider only permutations of $(x, y, z)$ given by:

$$
\pi=\left[\begin{array}{lll}
x & y & z \\
y & z & x
\end{array}\right] \quad \pi_{1}=\left[\begin{array}{lll}
x & y & z \\
x & z & y
\end{array}\right]
$$

Note that all other permutations can be derived by the given two ones.

Theorem 4. Orthogonal preserving $Q S O$, given in $S^{2}$, can be divided into three non-conjugate classes

$$
K_{1}=\left\{V_{\alpha, \beta, \gamma}^{(1)}, V_{\alpha, \beta, \gamma}^{(5)}, V_{\alpha, \beta, \gamma}^{(3)}\right\}
$$




$$
\begin{aligned}
& K_{2}=\left\{V_{\alpha, \beta, \gamma}^{(4)}, V_{\alpha, \beta, \gamma}^{(6)}\right\} \\
& K_{3}=\left\{V_{\alpha, \beta, \gamma}^{(2)}\right\}
\end{aligned}
$$

Proof. Let us consider $V_{\alpha, \beta, \gamma}^{(1)}$. Then one has

$$
\begin{aligned}
T_{\pi}^{-1} V_{\alpha, \beta, \gamma}^{(1)} T_{\pi}(x, y, z)= & T_{\pi}^{-1} V_{\alpha, \beta, \gamma}^{(1)}(y, z, x) \\
= & \left(y^{2}+2(1-\alpha) y z+2(1-\gamma) y x, x^{2}+2 \gamma y x\right. \\
& \left.+2 \beta z x, z^{2}+2 \alpha y z+2(1-\beta) z x\right) \\
= & V_{1-\gamma, 1-\alpha, \beta}^{(5)}
\end{aligned}
$$

This means that $V_{\alpha, \beta, \gamma}^{(1)} \sim^{\pi} V_{1-\gamma, 1-\alpha, \beta}^{(5)}$.

Similarly, we have $T_{\pi}^{-1} V_{\alpha, \beta, \gamma}^{(5)} T_{\pi}(x, y, z)=V_{1-\gamma, \alpha, 1-\beta}^{(3)}$. Hence, $V_{\alpha, \beta, \gamma}^{(5)} \sim$ $V_{1-\gamma, \alpha, 1-\beta}^{(3)}$. By the same argument one finds $T_{\pi}^{-1} V_{\alpha, \beta, \gamma}^{(3)} T_{\pi}(x, y, z)=V_{\gamma, 1-\alpha, 1-\beta}^{(1)}$ which means $V_{\alpha, \beta, \gamma}^{(3)} \sim^{\pi} V_{\gamma, 1-\alpha, 1-\beta}^{(1)}$.

This implies that the operators $V_{\alpha, \beta, \gamma}^{(1)}, V_{\alpha, \beta, \gamma}^{(5)}, V_{\alpha, \beta, \gamma}^{(3)}$ are conjugate and we put them into one class denoted by $K_{1}$.

One can obtain that $V_{\alpha, \beta, \gamma}^{(2)} \sim^{\pi} V_{1-\gamma, \alpha, 1-\beta}^{(2)}$ and $V_{\alpha, \beta, \gamma}^{(4)} \sim^{\pi} V_{1-\gamma, 1-\alpha, \beta}^{(4)}$ and $V_{\alpha, \beta, \gamma}^{(6)} \sim^{\pi}$ $V_{\gamma, 1-\alpha, 1-\beta}^{(6)}$. Therefore we need to consider another permutation $\pi_{1}$

Consequently, one finds $V_{\alpha, \beta, \gamma}^{(2)} \sim^{\pi_{1}} V_{\gamma, 1-\beta, \alpha}^{(2)}, V_{\alpha, \beta, \gamma}^{(4)} \sim^{\pi_{1}} V_{1-\gamma, \beta, \alpha}^{(6)}$.

Thus by $K_{2}$ we denote the class containing $V_{\alpha, \beta, \gamma}^{(4)}$ and $V_{\alpha, \beta, \gamma}^{(6)}$ and by $K_{3}$ class containing only $V_{\alpha, \beta, \gamma}^{(2)}$. This completes the proof.

Remark 3. One can see that the operator $V_{\alpha, \beta, \gamma}^{(2)}$ is a Volterra QSO, and its dynamics investigated in [3]. From the results of [25,29] one can conclude that even dynamics of Volterra QSO is very complicated. We note that if $\alpha, \beta, \gamma \in\{0,1\}$ then dynamics of operators taken from the classes $K_{1}$ and $K_{2}$ were investigated in [15-17]. In [4,30] certain general properties of dynamics of permuted Volterra QSO were studied.

Therefore, we propose the following

Problem 1. Investigate limiting behavior of the operators taken from the classes $K_{1}$ and $K_{2}$ (for any values of $\alpha, \beta, \gamma \in(0,1)$ ).

In general setting, we can also define orthogonal preserving condition. Namely, we call a QSO given by (3.4) orthogonal preserving if $V(\mu) \perp V(v)$ whenever $\mu \perp v$, where $\mu, \nu \in S(E, \mathcal{F})$. Taking into account Remark 1 we can formulate the following 
Conjecture 1. Let $V$ be a QSO given by (3.4). Then $V$ is orthogonal preserving if and only if there is a measurable automorphism $\alpha: E \rightarrow E\left(\right.$ i.e. $\left.\alpha^{-1}(\mathcal{F}) \subset \mathcal{F}\right)$ and a Volterra QSO $V_{0}$ such that $V \mu=V_{0}\left(\mu \circ \alpha^{-1}\right)$.

\section{ASSOCIATIVITY OF ORTHOGONALITY PRESERVING QSO}

In this section we state basic definitions and properties of genetics algebras.

Let $V$ be a QSO and suppose that $\mathbf{x}, \mathbf{y} \in \mathbb{R}^{m}$ are arbitrary vectors, we introduce a multiplication rule on $\mathbb{R}^{m}$ by

$$
\mathbf{x} \circ \mathbf{y}=\frac{1}{4}(V(\mathbf{x}+\mathbf{y})-V(\mathbf{x}-\mathbf{y}))
$$

This multiplication can be written as follows:

$$
(\mathbf{x} \circ \mathbf{y})_{k}=\sum_{i, j=1}^{m} P_{i j, k} x_{i} y_{j}
$$

where $\mathbf{x}=\left(x_{1}, \ldots, x_{m}\right), \mathbf{y}=\left(y_{1}, \ldots, y_{m}\right) \in \mathbb{R}^{m}$.

The pair $\left(\mathbb{R}^{m}, \circ\right)$ is called genetic algebra. We note the this algebra is commutative, i.e. $\mathbf{x} \circ \mathbf{y}=\mathbf{y} \circ \mathbf{x}$. Certain algebraic properties of such kind of algebras were investigated in $[9,28]$. In general, the genetic algebra no need to be associative. Therefore, we introduce the following

Definition 4. A QSO $V$ is called associative if the corresponding multiplication given by (5.2) is associative, i.e.

$$
(\mathbf{x} \circ \mathbf{y}) \circ \mathbf{z}=\mathbf{x} \circ(\mathbf{y} \circ \mathbf{z})
$$

hold for all $\mathbf{x}, \mathbf{y}, \mathbf{z} \in \mathbb{R}^{m}$.

In this section we are going to find associative orthogonal preserving QSO. According to the previous section, we have only three classes of OP QSO. Now we are interested whether these operators will be associative. Note that associativity of some classes of QSO has been investigated in $[2,19]$.

Theorem 5. The QSO $V_{\alpha, \beta, \gamma}^{(2)}$ is associative if and only if one of the following conditions are satisfied:

$$
\begin{array}{lll}
\text { (1) } \alpha=0, & \beta=0, & \gamma=0 \\
\text { (2) } \alpha=1, & \beta=0, & \gamma=0 \\
\text { (3) } \alpha=0, & \beta=1, & \gamma=1 \\
\text { (4) } \alpha=1, & \beta=1, & \gamma=1 \\
\text { (5) } \alpha=0, & \beta=1, & \gamma=0
\end{array}
$$


Proof. To show the associativity we will check the equality (5.3), which can be rewritten as follows:

$$
\begin{aligned}
\sum_{i, j=1}^{3} P_{i j, u} x_{i}\left(\sum_{m, k=1}^{3} P_{m k, j} y_{m} z_{k}\right) & \\
= & \sum_{i, j=1}^{3} P_{i j, u}\left(\sum_{m, k=1}^{3} P_{m k, i} x_{m} y_{k}\right) z_{j} \quad u=1,2,3
\end{aligned}
$$

where we have used the following equalities

$$
\begin{aligned}
(x \circ(y \circ z))_{u} & =\sum_{i, j=1}^{3} P_{i j, u} x_{i}\left(\sum_{m, k=1}^{3} P_{m k, j} y_{m} z_{k}\right) \\
((x \circ y) \circ z)_{u} & =\sum_{i, j=1}^{3} P_{i j, u}\left(\sum_{m, k=1}^{3} P_{m k, i} x_{m} y_{k}\right) z_{j} \quad u=1,2,3
\end{aligned}
$$

For $V_{\alpha, \beta, \gamma}^{(2)}$ the equality (5.4) can be written as follows:

$$
\begin{aligned}
x_{1} & \left(y_{1} z_{1}+\alpha y_{1} z_{2}+\gamma y_{1} z_{3}+\alpha y_{2} z_{1}+\gamma y_{3} z_{1}\right) \\
& +\alpha x_{1}\left((1-\alpha) y_{1} z_{2}+(1-\alpha) y_{2} z_{1}+y_{2} z_{2}+\beta y_{2} z_{3}+\beta y_{3} z_{2}\right) \\
& +\gamma x_{1}\left((1-\gamma) y_{1} z_{3}+(1-\beta) y_{2} z_{3}+(1-\gamma) y_{3} z_{1}+(1-\beta) y_{3} z_{2}+y_{3} z_{3}\right) \\
& +\alpha x_{2}\left(y_{1} z_{1}+\alpha y_{1} z_{2}+\gamma y_{1} z_{3}+\alpha y_{2} z_{1}+\gamma y_{3} z_{1}\right) \\
& +\gamma x_{3}\left(y_{1} z_{1}+\alpha y_{1} z_{2}+\gamma y_{1} z_{3}+\alpha y_{2} z_{1}+\gamma y_{3} z_{1}\right) \\
= & z_{1}\left(x_{1} y_{1}+\alpha x_{1} y_{2}+\gamma x_{1} y_{3}+\alpha x_{2} y_{1}+\gamma x_{3} y_{1}\right) \\
& +\alpha z_{2}\left(x_{1} y_{1}+\alpha x_{1} y_{2}+\gamma x_{1} y_{3}+\alpha x_{2} y_{1}+\gamma x_{3} y_{1}\right) \\
& +\gamma z_{3}\left(x_{1} y_{1}+\alpha x_{1} y_{2}+\gamma x_{1} y_{3}+\alpha x_{2} y_{1}+\gamma x_{3} y_{1}\right) \\
& +\alpha z_{1}\left((1-\alpha) x_{1} y_{2}+(1-\alpha) x_{2} y_{1}+x_{2} y_{2}+\beta x_{2} y_{3}+\beta x_{3} y_{2}\right) \\
& +\gamma z_{1}\left((1-\gamma) x_{1} y_{3}+(1-\beta) x_{2} y_{3}+(1-\gamma) x_{3} y_{1}+(1-\beta) x_{3} y_{2}+x_{3} y_{3}\right) ; \\
(1- & \alpha) x_{1}\left((1-\alpha) y_{1} z_{2}+(1-\alpha) y_{2} z_{1}+y_{2} z_{2}+\beta y_{2} z_{3}+\beta y_{3} z_{2}\right) \\
& +(1-\alpha) x_{2}\left(y_{1} z_{1}+\alpha y_{1} z_{2}+\gamma y_{1} z_{3}+\alpha y_{2} z_{1}+\gamma y_{3} z_{1}\right) \\
& +x_{2}\left((1-\alpha) y_{1} z_{2}+(1-\alpha) y_{2} z_{1}+y_{2} z_{2}+\beta y_{2} z_{3}+\beta y_{3} z_{2}\right) \\
& +\beta x_{2}\left((1-\gamma) y_{1} z_{3}+(1-\beta) y_{2} z_{3}+(1-\gamma) y_{3} z_{1}+(1-\beta) y_{3} z_{2}+y_{3} z_{3}\right) \\
& +\beta x_{3}\left((1-\alpha) y_{1} z_{2}+(1-\alpha) y_{2} z_{1}+y_{2} z_{2}+\beta y_{2} z_{3}+\beta y_{3} z_{2}\right) \\
= & (1-\alpha) z_{2}\left(x_{1} y_{1}+\alpha x_{1} y_{2}+\gamma x_{1} y_{3}+\alpha x_{2} y_{1}+\gamma x_{3} y_{1}\right) \\
& +(1-\alpha) z_{1}\left((1-\alpha) x_{1} y_{2}+(1-\alpha) x_{2} y_{1}+x_{2} y_{2}+\beta x_{2} y_{3}+\beta x_{3} y_{2}\right)
\end{aligned}
$$




$$
\begin{aligned}
&+z_{2}\left((1-\alpha) x_{1} y_{2}+(1-\alpha) x_{2} y_{1}+x_{2} y_{2}+\beta x_{2} y_{3}+\beta x_{3} y_{2}\right) \\
&+\beta z_{3}\left((1-\alpha) x_{1} y_{2}+(1-\alpha) x_{2} y_{1}+x_{2} y_{2}+\beta x_{2} y_{3}+\beta x_{3} y_{2}\right) \\
&+\beta z_{2}\left((1-\gamma) x_{1} y_{3}+(1-\beta) x_{2} y_{3}+(1-\gamma) x_{3} y_{1}+(1-\beta) x_{3} y_{2}+x_{3} y_{3}\right) ; \\
&(1-\gamma) x_{1}\left((1-\gamma) y_{1} z_{3}+(1-\beta) y_{2} z_{3}+(1-\gamma) y_{3} z_{1}+(1-\beta) y_{3} z_{2}+y_{3} z_{3}\right) \\
& \quad+(1-\beta) x_{2}\left((1-\gamma) y_{1} z_{3}+\left(1-\beta y_{2} z_{3}+(1-\gamma) y_{3} z_{1}+(1-\beta) y_{3} z_{2}+y_{3} z_{3}\right)\right. \\
& \quad+(1-\gamma) x_{3}\left(y_{1} z_{1}+\alpha y_{1} z_{2}+\gamma y_{1} z_{3}+\alpha y_{2} z_{1}+\gamma y_{3} z_{1}\right) \\
& \quad+(1-\beta) x_{3}\left((1-\alpha) y_{1} z_{2}+(1-\alpha) y_{2} z_{1}+y_{2} z_{2}+\beta y_{2} z_{3}+\beta y_{3} z_{2}\right) \\
& \quad+x_{3}\left((1-\gamma) y_{1} z_{3}+(1-\beta) y_{2} z_{3}+(1-\gamma) y_{3} z_{1}+(1-\beta) y_{3} z_{2}+y_{3} z_{3}\right) \\
&=(1-\gamma) z_{3}\left(x_{1} y_{1}+\alpha x_{1} y_{2}+\gamma x_{1} y_{3}+\alpha x_{2} y_{1}+\gamma x_{3} y_{1}\right) \\
& \quad+(1-\beta) z_{3}\left((1-\alpha) x_{1} y_{2}+(1-\alpha) x_{2} y_{1}+x_{2} y_{2}+\beta x_{2} y_{3}+\beta x_{3} y_{2}\right) \\
& \quad+(1-\gamma) z_{1}\left((1-\gamma) x_{1} y_{3}+(1-\beta) x_{2} y_{3}+(1-\gamma) x_{3} y_{1}+(1-\beta) x_{3} y_{2}+x_{3} y_{3}\right) \\
&+(1-\beta) z_{2}\left((1-\gamma) x_{1} y_{3}+(1-\beta) x_{2} y_{3}+(1-\gamma) x_{3} y_{1}+(1-\beta) x_{3} y_{2}+x_{3} y_{3}\right) \\
&++z_{3}\left((1-\gamma) x_{1} y_{3}+(1-\beta) x_{2} y_{3}+(1-\gamma) x_{3} y_{1}+(1-\beta) x_{3} y_{2}+x_{3} y_{3}\right) .
\end{aligned}
$$

Now equalizing the corresponding terms and simplifying the obtained expressions one gets:

$$
\begin{aligned}
& \beta(1-\beta)=0, \alpha(1-\gamma)=(\alpha-\gamma)(1-\beta), \alpha(1-\alpha)=0, \\
& \alpha(\gamma-\beta)=0, \gamma(1-\gamma)=0, \gamma(1-\beta)=0, \\
& (\beta-\gamma)(1-\alpha)=\beta(1-\gamma) .
\end{aligned}
$$

Solving these equations we get the desired equalities, which completes the proof.

Theorem 6. The operators $V_{\alpha, \beta, \gamma}^{(1)}$ and $V_{\alpha, \beta, \gamma}^{(4)}$ are not associative for any values of $\alpha, \beta, \gamma$.

Proof. Without loss of generality, we prove the theorem for $V_{\alpha, \beta, \gamma}^{(1)}$. Since for $V_{\alpha, \beta, \gamma}^{(4)}$ similar argument can be applied.

From (4.1), (5.1) with $\mathbf{x}=(0,0,1), \mathbf{y}=(0,1,0), \mathbf{z}=(1,0,0)$ we easily find

$$
\begin{aligned}
& (\mathbf{x} \circ \mathbf{y}) \circ \mathbf{z}=(0, \alpha(1-\beta), 1-\alpha(1-\beta)) \\
& \mathbf{x} \circ(\mathbf{y} \circ \mathbf{z})=(1-\alpha(1-\beta),(1-\beta) \alpha, 0)
\end{aligned}
$$

It is clear that if $\alpha \neq 1, \beta \neq 0$, then the operator $V_{\alpha, \beta, \gamma}^{(1)}$ is not associative.

Therefore, we suppose that $\alpha=1$ and $\beta=0$. Then from (4.1) one finds

$$
\begin{aligned}
& (\mathbf{x} \circ \mathbf{y})_{1}=x_{3} y_{3}+\gamma\left(x_{1} y_{3}+x_{3} y_{1}\right), \\
& (\mathbf{x} \circ \mathbf{y})_{2}=x_{2} y_{2}+x_{1} y_{2}+x_{2} y_{1}+x_{2} y_{3}+x_{3} y_{2}, \\
& (\mathbf{x} \circ \mathbf{y})_{3}=x_{3} y_{3}+(1-\gamma)\left(x_{1} y_{3}+x_{3} y_{1}\right) .
\end{aligned}
$$


Now taking the elements $\mathbf{x}=(1,1,0), \mathbf{y}=(1,1,0), \mathbf{z}=(1,0,1)$ we find

$$
\begin{aligned}
& (\mathbf{x} \circ \mathbf{y}) \circ \mathbf{z}=(1+\gamma, 6,1-\gamma) \\
& \mathbf{x} \circ(\mathbf{y} \circ \mathbf{z})=\left(2 \gamma-\gamma^{2}, 6,2-2 \gamma+\gamma^{2}\right) .
\end{aligned}
$$

One can see that $(\mathbf{x} \circ \mathbf{y}) \circ \mathbf{z} \neq \mathbf{x} \circ(\mathbf{y} \circ \mathbf{z})$, since $\gamma(1-\gamma)=1$ has not any real solution. Hence, $V_{1,0, \gamma}^{(1)}$ is not associative. This completes the proof.

\section{ACKNOWLEDGEMENT}

The authors are grateful to a referee for his useful suggestions which improved the style and contents of the paper.

\section{REFERENCES}

[1] S. Bernstein, "The solution of a mathematical problem concerning the theory of heredity," Ucheniye-Zapiski N.-I. Kaf. Ukr. Otd. Mat., vol. 1, pp. 83-115, 1924.

[2] N. Ganikhodjaev and H. Hisamuddin, "Associativity in inheritance or are there associative populations," Malaysian J. Sci., vol. 27, no. 2, pp. 131-136, 2008.

[3] R. Ganikhodzhaev, "Quadratic stochastic operators, Lyapunov functions and tournaments," Russ. Acad. Sci., Sb., Math., vol. 76, no. 2, pp. 489-506, 1992, doi: 10.1070/SM1993v076n02ABEH003423.

[4] R. Ganikhodzhaev and D. Eshmamatova, "Quadratic automorphisms of a simplex and the asymptotic behavior of their trajectories," Vladikavkaz. Mat. Zh., vol. 8, no. 2, pp. 12-28, 2006.

[5] R. Ganikhodzhaev, F. Mukhamedov, and U. Rozikov, "Quadratic stochastic operators and processes: results and open problems," Infin. Dimens. Anal. Quantum Probab. Relat. Top., vol. 14, no. 2, pp. 270-335, 2011, doi: 10.1142/S0219025711004365.

[6] J. Hofbauer, V. Hutson, and W. Jansen, "Coexistence for systems governed by difference equations of Lotka-Volterra type," J. Math. Biol., vol. 25, no. 2, pp. 553-570, 1987, doi: 10.1007/BF00276199.

[7] J. Hofbauer and K. Sigmund, The theory of evolution and dynamical systems, 1st ed., ser. London Mathematical Society Student Texts. Cambridge: Cambridge University Press, 1988, vol. 7.

[8] A. Lotka, "Undamped oscillations derived from the law of mass action," J. Amer. Chem. Soc., vol. 42, pp. 1595-1599, 1920, doi: 10.1021/ja01453a010.

[9] Y. Lyubich, Mathematical structures in population genetics. Berlin: Springer-Verlag, 1992.

[10] R. May, "Simple mathematical models with very complicated dynamics," Nature, vol. 261, pp. 459-467, 1976.

[11] R. May and G. Oster, "Bifurcations and dynamic complexity in simple ecological models," Am. Nat., vol. 110, pp. 573-599, 1976.

[12] F. Mukhamedov, I. Qaralleh, and W. Rozali, "On $\xi^{(a)}$-quadratic stochastic operators on 2D simplex," Sains Malaysiana, vol. 43, no. 8, pp. 1275-1281, 2014.

[13] F. Mukhamedov and M. Saburov, "On homotopy of volterrian quadratic stochastic operator," Appl. Math. Inform. Sci., vol. 4, no. 1, pp. 47-62, 2010.

[14] F. Mukhamedov and M. Saburov, "On dynamics of Lotka-Volterra type operators," Bull. Malays. Math. Sci. Soc. (2), vol. 37, no. 1, pp. 59-64, 2014.

[15] F. Mukhamedov, M. Saburov, and A. Jamal, "On dynamics of $\xi^{s}$-quadratic stochastic operators," Inter. Jour. Modern Phys.: Conf. Ser., vol. 9, pp. 299-307, 2012, doi: 10.1142/S2010194512005351. 
[16] F. Mukhamedov, M. Saburov, and I. Qaralleh, "Classification of $\xi^{(s)}$-quadratic stochastic operators on 2D simplex," J. Phys.: Conf. Ser., vol. 435, p. 012003, 2013.

[17] F. Mukhamedov, M. Saburov, and I. Qaralleh, "On $\xi^{(s)}$-quadratic stochastic operators on two dimensional simplex and their behaviors," Abst. Appl. Anal., vol. 2013, p. 12, 2013, doi: 10.1155/2013/942038.

[18] S. Narendra, C. Samaresh, and W. Elliott, "On the Volterra and other nonlinear moldes of interacting populations," Rev. Mod. Phys., vol. 43, pp. 231-276, 1971, doi: 10.1103/RevModPhys.43.231.

[19] N. Narziev, "On subalgebras of genetic algebras arising on mathematical models of population genetics," Malay. J. Math. Sci., vol. 2, pp. 171-181, 2010.

[20] M. Plank, "Hamiltonian structures for the $n$-dimensional Lotka-Volterra equations," J. Math. Phys., vol. 36, no. 7, pp. 3520-3543, 1995.

[21] U. Rozikov and S. Nazir, "Separable quadratic stochastic operators," Lobachevskii Jour. Math., vol. 31, no. 3, pp. 215-221, 2010, doi: 10.1134/S1995080210030030.

[22] U. Rozikov and A. Zada, "On l-Volterra quadratic stochastic operators," Dokl. Math., vol. 79, no. 1, pp. 32-34, 2009, doi: 10.1134/S1064562409010104.

[23] Y. Takeuchi, Global dynamical properties of Lotka-Volterra systems. Singapore: World Scientific, 1996.

[24] S. Ulam, A collection of mathematical problems, ser. Interscience Tracts in Pure and Applied Mathematics. New York: Interscience Publishers, 1960, vol. 8.

[25] S. Vallander, "On the limit behavior of iteration sequence of certain quadratic transformations," Sov. Math. Dokl., vol. 13, pp. 123-126, 1972.

[26] V. Volterra, "Lois de fluctuation de la population de plusieurs espèces coexistant dans le même milieu," Association Française Lyon, vol. 1926, pp. 96-98, 1927.

[27] V. Volterra, Leçons sur la théorie mathématique de la lutte pour la vie, ser. Cahiers scientifiques fasc. Paris: Gauthiers-Villars, 1931, vol. 7.

[28] A. Worz-Busekros, Algebras in Genetics, ser. Lecture Notes in Biomathematics. Berlin: Springer, 1980, vol. 36.

[29] M. Zakharevich, "On the behaviour of trajectories and the ergodic hypothesis for quadratic mappings of a simplex," Russ. Math. Surv., vol. 33, no. 6, pp. 265-266, 1978, doi: 10.1070/RM1978v033n06ABEH003890.

[30] U. Zhamilov and U. Rozikov, "The dynamics of strictly non-Volterra quadratic stochastic operators on the 2-simplex," Sbornik: Math., vol. 200, no. 9, pp. 1339-1352, 2009, doi: 10.1070/SM2009v200n09ABEH004039.

Authors' addresses

\section{Farrukh Mukhamedov}

International Islamic University Malaysia, Faculty of Science, Department of Computational \& Theoretical Sciences, P.O. Box, 141, 25710, Kuantan, Pahang, Malaysia

E-mail address: far75m@yandex.ru; farrukh_meiium.edu.my

Muhammad Hafizuddin Bin Mohd Taha

International Islamic University Malaysia, Faculty of Science, Department of Computational \& Theoretical Sciences, P.O. Box, 141, 25710, Kuantan, Pahang, Malaysia 